\title{
LightCycler SeptiFast assay as a tool for the rapid diagnosis of sepsis in patients during antimicrobial therapy
}

Rapid aetiological diagnosis and early administration of adequate antimicrobial therapy soon after a patient's arrival at the hospital are crucial for the successful management of sepsis and septic shock (Dombrovskiy et al., 2007; Raghavan \& Marik, 2006). Routine aetiological diagnosis of sepsis relies on standard culture methods that take at least $24 \mathrm{~h}$ or more to give the initial information. Therefore, quick identification of pathogens causing sepsis within the clinically relevant time frame should be based on state-of-the-art molecular methods that target bacterial and fungal DNA (Abdul-Redha et al., 2007; Breitkopf et al., 2005; Dombrovskiy et al., 2007). In this letter, we describe the clinical utility of the first standardized, CE-certified, multiplex real-time PCR assay for the molecular diagnosis of sepsis that has been approved for in vitro diagnostic use (LightCycler SeptiFast assay; Roche Diagnostics). The aim of our preliminary study was to determine the additional diagnostic value of the LightCycler SeptiFast assay, which enables detection of DNA from 25 human pathogens (Gram-positive and Gram-negative bacteria as well as fungi) in the blood of patients with suspected sepsis even after empirical antimicrobial therapy has been started. The study enrolled 36 patients (a total of 39 samples) with a clinical diagnosis of sepsis that were treated at the University Hospital for Infectious Diseases, Zagreb, and Zagreb University Clinical Center in Croatia. Seventeen patients were hospitalized in the intensive care unit (ICU), nine patients outside the ICU and ten patients in the Department of Haematology following bone marrow or peripheral blood stem cell transplantation (BSCT). All patients enrolled in the study were already receiving empirical antimicrobial therapy at the time of testing. Blood cultures were taken from all patients as well. Thirteen out of 39 (33\%) samples tested positive with the SeptiFast assay for bacterial or fungal DNA (Table 1). Gramnegative bacterial DNA was detected in 11 of 13 samples (Klebsiella pneumoniael oxytoca $n=4$; Escherichia coli $n=3$; Pseudomonas aeruginosa $n=4)$. Gram-positive bacterial DNA (Enterococcus faecium $n=1$;

Streptococcus pneumoniae $n=1$ ) was detected in two patients with polymicrobial sepsis (in combination with K. pneumoniael oxytoca in both patients). Aspergillus fumigatus DNA was detected in two patients with fever and pulmonary infiltrates. Additional SeptiFast-positive results (blood culture-negative) were obtained in nine of 36 patients. Four of 39 samples $(10.3 \%)$ were negative by the SeptiFast assay but positive by culture and those results were interpreted as false-negatives. Four of 39 samples were positive by both the SeptiFast assay and culture. Twenty-two samples tested negative by both techniques. We observed one discordant result between the SeptiFast assay and culture (Enterobacter cloacae was detected by culture and $K$. pneumoniae/oxytoca by SeptiFast assay). Incorrect interpretation of Enterobacter aerogenes/cloacae as K. pneumoniae/oxytoca due to the similarities of internal transcribed spacer regions of the target micro-organisms was also described by the manufacturer of the assay ( $4.2 \%$ error rate in the evaluation study). The SeptiFast assay does not detect Actinobacillus sp. DNA, but this microorganism was detected in one patient by culture only. In the group of 10 haematological patients, SeptiFast results were positive for six of the 10 patients (60\%), whereas blood cultures were positive in only two out of 10 patients $(20 \%)$. The declared analytical sensitivity of the SeptiFast assay ranges between 30 and 100 c.f.u. (depending on the micro-organism). The SeptiFast assay provides a rapid identification of the causative microorganism within $6 \mathrm{~h}$ (3 h of technician hands-on time) whereas blood cultures usually require 2 days for Gram-staining results and a total of 3 days for the species to be identified. However, the use of the SeptiFast assay is limited to well-established molecular laboratories, requires excellent technical skills and is very expensive compared to culture. Additionally, the SeptiFast assay cannot provide information regarding the antibiotic susceptibility of micro-organisms. We conclude that the SeptiFast assay is a clinically valuable add-on to conventional culture methods for rapid aetiological diagnosis of sepsis in patients where empirical antimicrobial therapy has already been started and pretreatment blood cultures were negative. This assay showed particular sensitivity in haematological patients following BSCT,

Table 1. Pathogens detected by the SeptiFast assay according to study groups

ICU, intensive care unit.

\begin{tabular}{|lcrc|}
\hline Study groups & Gram-positive bacteria & Gram-negative bacteria & Fungi \\
\hline Patients from the ICU & 0 & 3 & 1 \\
Patients outside the ICU & $1^{*}$ & 3 & 4 \\
Haematological patients & $1^{*}$ & 5 & 4 \\
Total & 2 & 11 & 7 \\
\end{tabular}

${ }^{\star}$ Two patients with polymicrobial sepsis (in combination with Klebsiella pneumoniae/oxytoca in both patients). 
where the administration of early antimicrobial therapy for febrile neutropenia is crucial.

\section{Acknowledgements}

This work was in part supported by Ministry of Science, Education and Sports of the Republic of Croatia grants 143-10801160097 and 143-0000000-0117 to S. Ž. L. and A. V., respectively.

Adriana Vince, ${ }^{1}$ Snježana

Židovec Lepej, ${ }^{1}$ Bruno Baršić, ${ }^{1}$

Davorka Dušek, ${ }^{1}$ Zdravko Mitrović, ${ }^{2}$

\section{Ranka Serventi-Seiwerth ${ }^{2}$} and Boris Labar ${ }^{2}$

${ }^{1}$ University Hospital for Infectious Diseases "Dr Fran Mihaljević", Mirogojska 8, 10000 Zagreb, Croatia

${ }^{2}$ Zagreb University Hospital Center, Kišpatićeva 12, 10000 Zagreb, Croatia

Correspondence: Snježana Židovec Lepej (Snjezana.Zidovec.Lepej@bfm.hr)

Abdul-Redha, R. J., Balslew, U., Christensen, J. J. \& Kemp, M. (2007). Globicatella sanguinis bacteraemia identified by partial $16 \mathrm{~S}$ rRNA gene sequencing. Scand J Infect Dis 39, 745-748.

Breitkopf, C., Hammel, D., Scheld, H. H., Peters, G. \& Becker, K. (2005). Impact of a molecular approach to improve the microbiological diagnostics of infective heart valve endocarditis. Circulation 111, 1415-1421.

Dombrovskiy, V. Y., Martin, A. A., Sunderram, J. \& Paz, H. L. (2007). Rapid increase in hospitalization and mortality rates for severe sepsis in the United States: a trend analysis from 1993-2003. Crit Care Med 35, 1244-1250.

Raghavan, M. \& Marik, P. E. (2006)

Management of sepsis during the early "golden hours". J Emerg Med 31, 185-199. 